\title{
A MULTIVARIATE "INV" HOOK FORMULA FOR FORESTS
}

\author{
FLORENT HIVERT AND VICTOR REINER
}

To Dennis Stanton on his 60th birthday

\begin{abstract}
Björner and Wachs provided two $q$-generalizations of Knuth's hook formula counting linear extensions of forests: one involving the major index statistic, and one involving the inversion number statistic. We prove a multivariate generalization of their inversion number result, motivated by specializations related to the modular invariant theory of finite general linear groups.
\end{abstract}

\section{INTRODUCTION}

This paper concerns formulas counting linear extensions of partial orders $P$ on the set $\{1,2, \ldots, n\}$ which are forests, in the sense that every element covers at most one other element. Recall that a permutation $w$ is a linear extension of the poset $P$ if the linear order $w_{1}<_{w} \ldots<_{w} w_{n}$ has the property that $i<_{P} j$ implies $i<_{w} j$. Denote by $\mathcal{L}(P)$ the set of all linear extensions of $P$. Knuth observed the following.

Theorem. (Knuth [7, §5.1.4, Exer. 20]) For any forest poset $P$ on $\{1,2, \ldots, n\}$, one has

$$
|\mathcal{L}(P)|=\frac{n !}{\prod_{i=1}^{n} h_{i}}
$$

where $h_{i}:=\left|P_{\geq i}\right|$ is the cardinality of the subtree $P_{\geq i}$ rooted at $i$.

Björner and Wachs [1] later gave two interesting $q$-generalizations of Knuth's result, both counting linear extensions according to certain statistics: the inversion number statistic inv, and the major index statistic maj. The following theorem rephrases a special case of the first of these results, relating to inv; see Remark 9.6 below for their second generalization.

Say that a forest poset $P$ is recursively labelled if the label set on each subtree $P_{\geq i}$ forms an interval in the integers, that is, $P_{\geq i}=\{a, a+1, \ldots, b-1, b\}$ for some integers $a=: \min \left(P_{\geq i}\right)$ and $b=: \max \left(P_{\geq i}\right)$. Define the inversion number $\operatorname{inv}(P)$ to be the number of pairs $i<_{\mathbb{Z}} j$ for which $i>_{P} j$. For example, the following picture

1991 Mathematics Subject Classification. 05A15, 05A10.

Key words and phrases. hook formula, forests, moulds, binary search, free quasisymmetric functions, Loday-Ronco algebra.

First author partially supported by grant ANR-06-BLAN-0380. Second author partially supported by NSF grant DMS-0601010. The second author also thanks A. Lascoux, J.-C. Novelli, and J.-Y. Thibon of the Institut Gaspard Monge at the University of Marne-la-Vallée for their hospitality during part of this work. 
shows the Hasse diagram of a recursively labelled forest $P$ on $\{1,2, \ldots, 10\}$.

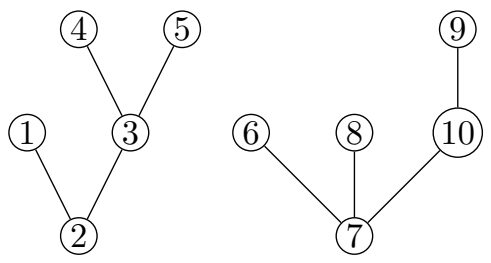

Here one has $P_{\geq 3}=\{3,4,5\}, P_{\geq 7}=\{6,7,8,9,10\}$, and

$$
\operatorname{inv}(P)=3=|\{(1,2),(6,7),(9,10)\}| .
$$

Lastly, define the $q$-analogues

$$
\begin{aligned}
{[n]_{q} } & :=1+q+q^{2}+\cdots+q^{n-1}, \\
{[n] !_{q}: } & =[n]_{q}[n-1]_{q}[n-2]_{q} \cdots[2]_{q}[1]_{q} .
\end{aligned}
$$

Theorem. (Björner and Wachs [1, Thm. 1.1])

Any recursively labelled forest $P$ on $\{1,2, \ldots, n\}$ has

$$
\sum_{w \in \mathcal{L}(P)} q^{\operatorname{inv}(w)}=q^{\operatorname{inv}(P)} \frac{[n] !_{q}}{\prod_{i=1}^{n}\left[h_{i}\right]_{q}} .
$$

Our goal is a multivariate generalization, Theorem 1.1 below. It is an identity within the field of rational functions $\mathbb{Q}(\mathbf{x}):=\mathbb{Q}\left(x_{1}, x_{2}, x_{3}, \ldots\right)$ in a sequence of indeterminates $x_{1}, x_{2}, x_{3}, \ldots$, related by a map $F$ sending $x_{i} \mapsto x_{i+1}$ that we call the Frobenius map. We introduce the following multivariate analogues of the positive integers $n$ and the factorial $n$ !:

$$
\begin{aligned}
{[1] } & :=x_{1} \\
{[n] } & :=[1]+F[1]+F^{2}[1]+\cdots+F^{n-1}[1] \\
& =x_{1}+x_{2}+\cdots+x_{n} \\
{[n] ! } & :=[n] \cdot F([n-1]) \cdot F^{2}([n-2]) \cdots F^{n-2}([2]) \cdot F^{n-1}([1]) \\
& =[n] \cdot F([n-1] !) .
\end{aligned}
$$

For example,

$$
[4] !=\left(x_{1}+x_{2}+x_{3}+x_{4}\right)\left(x_{2}+x_{3}+x_{4}\right)\left(x_{3}+x_{4}\right) x_{4} .
$$

After defining in Section 5 a weight $\operatorname{wt}(w)$ lying in $\mathbb{Q}(\mathbf{x})$ for each permutation $w$, we prove in Section 7 the following main result.

Theorem 1.1. Any recursively labelled forest $P$ on $\{1,2, \ldots, n\}$ has

$$
L(P):=\sum_{w \in \mathcal{L}(P)} \operatorname{wt}(w)=\frac{[n] !}{\prod_{i=1}^{n} F^{\min \left(P_{\geq i}\right)-1}\left[h_{i}\right]} .
$$

Section 8 explains why Theorem 1.1 becomes (1.1) upon applying the following $q$-specialization map to both sides:

$$
\begin{aligned}
\mathbb{Q}\left(x_{1}, x_{2}, \ldots\right) & \stackrel{\mathrm{sp}_{q}}{\longrightarrow} \mathbb{Q}(q) \\
x_{i} & \longmapsto q^{i-1}-q^{i} .
\end{aligned}
$$




\section{INVARIANT THEORY MOTIVATION}

Aside from the Björner-Wachs inv formula, a second motivation for Theorem 1.1 stems from previous joint work in invariant theory with D. Stanton [11]. The reader interested mainly in Theorem 1.1 and its connection to the work of Björner and Wachs can safely skip this explanation of the invariant-theoretic connection.

There are two special cases of Theorem 1.1 that turn out be equivalent to results from [11, namely the cases where either

(a) $P$ is a disjoint union of chains, each labelled by a contiguous interval of integers in increasing order [11, Theorem 8.6], or

(b) $P$ is a hook poset [11, Eqn. (6.1) and (11.1)], having

$$
1>_{P} 2>_{P} \cdots>_{P} m<_{P} m+1<_{P} \cdots<_{P} n-1<_{P} n .
$$

The story from [1] begins with $G:=G L_{n}\left(\mathbb{F}_{q}\right)$ acting by linear substitutions of variables on the polynomial algebra $S(q):=\mathbb{F}_{q}\left[x_{1}, \ldots, x_{n}\right]$. A well-known result of L.E. Dickson asserts that the $G$-invariant subalgebra $S(q)^{G}$ is again a polynomial algebra.

For each composition $\alpha=\left(\alpha_{1}, \ldots, \alpha_{\ell}\right)$ of $n$, one associates two families of $G$ representations $V(q)$ over $\mathbb{F}_{q}$, described below. For both of these representations $V(q)$, the graded intertwiner spaces

$$
M(q):=\operatorname{Hom}_{\mathbb{F}_{q} G}(V(q), S(q))
$$

were shown in [1] to be free modules over $S(q)^{G}$, and explicit formulas were given for the degrees of their $S(q)^{G}$-basis elements, or equivalently for the Hilbert series

$$
\operatorname{Hilb}_{q}(t):=\operatorname{Hilb}\left(M(q) / S(q)_{+}^{G} M(q), t\right) .
$$

These Hilbert series come from generating functions in $\mathbb{Q}(\mathbf{x})$ by applying the following $(q, t)$-specialization map

$$
\begin{aligned}
\mathbb{Q}\left(x_{1}, x_{2}, \ldots\right) & \stackrel{\mathrm{sp}_{q, t}}{\longmapsto} \mathbb{Q}(q) \\
x_{i} & \longmapsto t^{q^{i-1}}-t^{q^{i}}
\end{aligned}
$$

which is less drastic than the specialization in (1.4).

The first family of $G$-representations $V(q)$ associated to $\alpha$ is the permutation module for $G$ acting on $\alpha$-flags of $\mathbb{F}_{q}$-subspaces

$$
0 \subset V_{\alpha_{1}} \subset V_{\alpha_{1}+\alpha_{2}} \subset V_{\alpha_{1}+\alpha_{2}+\alpha_{3}} \subset \cdots \subset \mathbb{F}_{q}^{n}
$$

where $\operatorname{dim}_{\mathbb{F}_{q}} V_{i}=i$. For this family one has $\operatorname{Hilb}_{q}(t)=\operatorname{sp}_{q, t} L(P)$ where the poset $P$ is as described in case (a) above, when the chains have lengths $\alpha_{1}, \ldots, \alpha_{\ell}$.

The second family of $G$-representations $V(q)$ associated to $\alpha$ is the homology with $\mathbb{F}_{q}$-coefficients of the subcomplex of the Tits building generated by the faces indexed by $\alpha$-flags. For this family one has $\operatorname{Hilb}_{q}(t)=\operatorname{sp}_{q, t} L(P)$ where the poset $P$ is the rim hook poset $P$ for $\alpha$, having increasing chains of lengths $\alpha_{1}, \ldots, \alpha_{\ell}$, generalizing the $\alpha=\left(1^{m}, n-m\right)$ case described in (b) above.

In fact, for either of these classes of posets $P$ associated to $\alpha$, the more drastic $q$ specialization $\operatorname{sp}_{q} L(P)$ was shown to have two parallel representation-theoretic and invariant-theoretic interpretations. On one hand, $\operatorname{sp}_{q} L(P)=\operatorname{dim}_{\mathbb{F}_{q}} V(q)$. On the other hand, both classes of $\mathbb{F}_{q} G$-modules $V(q)$ have $(q=1)$ analogous $\mathbb{Z} W$-module counterparts $V$ where $W=\mathfrak{S}_{n}$ is the symmetric group. In particular, when one 
regards $W$ acting on $S:=\mathbb{Z}\left[x_{1}, \ldots, x_{n}\right]$ by permuting the variables, so that $S^{W}$ is the ring of symmetric polynomials, one finds that the graded intintertwiner space

$$
M:=\operatorname{Hom}_{\mathbb{Z} W}(V, S)
$$

turns out to be a free $S^{W}$-module, and that

$$
\operatorname{Hilb}\left(M / S_{+}^{W} M, q\right)=\operatorname{sp}_{q} L(P) .
$$

\section{Binomial coefficient and Pascal Recurrence}

Definition 3.1. (cf. [11, (1.2)]) Define a multivariate analogue of a binomial coefficient

$$
\left[\begin{array}{l}
n \\
k
\end{array}\right]:=\frac{[n] !}{[k] ! \cdot F^{k}([n-k] !)} .
$$

It is an easy exercise in the definitions (1.3) to deduce the following analogue of the usual Pascal recurrence.

Proposition 3.2. (cf. [11, 1st equation in (4.2)])

$$
\left[\begin{array}{l}
n \\
k
\end{array}\right]=F\left[\begin{array}{l}
n-1 \\
k-1
\end{array}\right]+\frac{F[k] !}{[k] !} \cdot F\left[\begin{array}{c}
n-1 \\
k
\end{array}\right] .
$$

\section{The Weight of A SUbSet}

The Pascal recurrence leads to an interpretation of the binomial coefficient as a sum over certain partitions (cf. [11, (5.1)]). For our purpose, it is better to rephrase it as weight $\operatorname{wt}(S)$ defined for sets $S$ of positive integers: a $k$-element set

$$
S=\left\{i_{1}>i_{2}>\cdots>i_{k}\right\}
$$

of positive integers, indexed in decreasing order, bijects with a partition $\lambda$ whose Ferrers diagram fits inside a $k \times(n-k)$ rectangle:

$$
\lambda(S):=\left(i_{1}, i_{2}, \ldots, i_{k}\right)-(k, k-1, \ldots, 2,1) .
$$

We thus re-encode the definition in [11, (5.1)] as follows.

Definition 4.1. For a $k$-element set $S$ of positive integers indexed as in (4.1), define

$$
\operatorname{wt}(S):=\frac{\prod_{j=1}^{k} F^{i_{j}-1}[j]}{[k] !}=\prod_{j=1}^{k} \frac{F^{i_{j}-1}[j]}{F^{k-j}[j]} .
$$

Example 4.2. For $k=5$, the set $S=\{9,7,6,4,2\}$ has weight

$$
\operatorname{wt}(S)=\frac{F^{8}[1] F^{6}[2] F^{5}[3] F^{3}[4] F^{1}[5]}{[5] !} .
$$

Using the notation

$$
S+1:=\{i+1: i \in S\}
$$

one can also define this weight recursively as follows:

$$
\operatorname{wt}(S):= \begin{cases}1 & \text { if } S=\emptyset \\ \frac{F[k] !}{[k] !} F \operatorname{wt}(\hat{S}) & \text { if } 1 \notin S \text { and } S=\hat{S}+1 \\ F \operatorname{wt}(\hat{S}) & \text { if } 1 \in S \text { and } S=\{1\} \cup(\hat{S}+1) .\end{cases}
$$


Proposition 4.3. (cf. [11, Theorem 5.3])

$$
\left[\begin{array}{l}
n \\
k
\end{array}\right]=\sum_{S} \mathrm{wt}(S)
$$

where the sum runs over all subsets $S$ of cardinality $k$ of $\{1, \ldots n\}$.

Proof. Induct on $n$ with trivial base case $n=0$. In the inductive step, the sum in the right hand side of the proposition decomposes as two subsums

$$
\sum_{1 \in S} \mathrm{wt}(S)+\sum_{1 \notin S} \mathrm{wt}(S)
$$

which correspond to the two terms in the Pascal recurrence, Proposition 3.2 . Using the recursive definition (4.3) then completes the inductive step.

\section{The Weight of a PERMUtATION VIA RECURSion}

We wish to extend the definition of the weight $\operatorname{wt}(S)$ for a set $S$ to a weight $\operatorname{wt}(w)$ for permutations $w$ in $\mathfrak{S}_{n}$, defined recursively, following [11, §8].

Definition 5.1. 11, Definition 8.1] Given $w=\left(w_{1}, w_{2}, \ldots, w_{n}\right)$ in $W:=\mathfrak{S}_{n}$, let $k:=w_{1}-1$, so that $0 \leq k \leq n-1$ and $w_{1}=k+1$. Regarding $w$ as a shuffle of its restrictions to the alphabets $[1, k]$ and $[k+1, n]$, one can factor it uniquely

$$
w=u \cdot a \cdot b
$$

with $u$ a minimum-length coset representative of $u W_{J}$ for the parabolic or Young subgroup

$$
\begin{aligned}
W_{J} & :=\mathfrak{S}_{[1, k]} \times \mathfrak{S}_{[k+1, n]} \\
& \cong \mathfrak{S}_{k} \times \mathfrak{S}_{n-k}
\end{aligned}
$$

and where $a, b$ lie in $\in \mathfrak{S}_{[1, k]}, \mathfrak{S}_{[k+1, n]}$, respectively.

Since $u$ is a shuffle of the increasing sequences $(1,2, \ldots, k),(k+1, k+2, \ldots, n)$, it can be encoded via the set

$$
S(u):=\left\{u^{-1}(k)>u^{-1}(k-1)>\cdots>u^{-1}(2)>u^{-1}(1)\right\} .
$$

Since $w_{1}=k+1$ implies $b(k+1)=k+1$, the permutation $b$ in $\mathfrak{S}_{[k+1, n]}$ actually lies in the subgroup $\mathfrak{S}_{[k+2, n]}$ that fixes $k+1$, isomorphic to $\mathfrak{S}_{n-k-2}$. Denote by $\hat{b}$ the corresponding element of $\mathfrak{S}_{n-k-2}$.

Now define $\operatorname{wt}(w)$ recursively by saying that the identity element $e$ in $\mathfrak{S}_{0}$ has $\operatorname{wt}(e):=1$, and otherwise

$$
\operatorname{wt}(w):=\operatorname{wt}(S(u)) \cdot \operatorname{wt}(a) \cdot F^{k+1}(\operatorname{wt}(\hat{b})) .
$$

Note that since $k=w_{1}-1$, the integer 1 is never in $S(u)$. Therefore writing $S(u)=\hat{S}(u)+1$ for a $k$-element subset of $\{1,2, \ldots, n-1\}$, one can use (4.3) to rewrite (5.3) as

$$
\operatorname{wt}(w):=\frac{F[k] !}{[k] !} F(\operatorname{wt}(\hat{S}(u))) \cdot \operatorname{wt}(a) \cdot F^{k+1}(\operatorname{wt}(\hat{b})) .
$$


Example 5.2. For $n=9$, consider within $\mathfrak{S}_{9}$ the permutation

$$
\begin{aligned}
w & =\left(\begin{array}{lllllllll}
1 & \underline{2} & 3 & \underline{4} & 5 & \frac{6}{6} & \frac{\mathbf{7}}{8} & 8 & \frac{9}{6} \\
6 & \mathbf{2} & 9 & \mathbf{1} & 7 & \mathbf{5} & \mathbf{3} & 8 & \mathbf{4}
\end{array}\right) \\
& =\underbrace{\left(\begin{array}{lllllllll}
1 & 2 & 3 & \frac{4}{2} & 5 & \frac{6}{7} & \mathbf{7} & 8 & \frac{9}{6} \\
6 & \mathbf{1} & 7 & \mathbf{2} & 8 & \mathbf{3} & \mathbf{4} & 9 & \mathbf{5}
\end{array}\right)}_{u} \cdot \underbrace{\left(\begin{array}{lllll}
1 & 2 & 3 & 4 & 5 \\
2 & 1 & 5 & 3 & 4
\end{array}\right)}_{a} \cdot \underbrace{\left(\begin{array}{lllll}
6 & 7 & 8 & 9 \\
6 & 9 & 7 & 8
\end{array}\right)}_{b}
\end{aligned}
$$

One has $k=w_{1}-1=6-1=5$ here, and note that $b(6)=6$, with

$$
\hat{b}=\left(\begin{array}{lll}
1 & 2 & 3 \\
3 & 1 & 2
\end{array}\right) .
$$

Since the values $\{1,2,3,4,5(=k)\}$ occur in positions $S(u)=\{9,7,6,4,2\}$ of $u$ or $w$, one has that

$$
\begin{aligned}
\operatorname{wt}(w) & =\operatorname{wt}(\{9,7,6,4,2\}) \cdot \operatorname{wt}(a) \cdot F^{6} \operatorname{wt}(\hat{b}) \\
& =\frac{F^{8}[1] F^{6}[2] F^{5}[3] F^{3}[4] F[5]}{[5] !} \cdot \operatorname{wt}(a) \cdot F^{6} \operatorname{wt}(\hat{b}) .
\end{aligned}
$$

Finishing the recursive computation, one finds

$$
\begin{gathered}
\operatorname{wt}(a)=\operatorname{wt}(21534)=\frac{\left(x_{4}+x_{5}\right) x_{2} x_{5}}{\left(x_{3}+x_{4}\right) x_{1} x_{4}}, \quad \operatorname{wt}(b)=\operatorname{wt}(312)=\frac{\left(x_{2}+x_{3}\right) x_{3}}{\left(x_{1}+x_{2}\right) x_{2}}, \\
\operatorname{wt}(\{9,7,6,4,2\})=\frac{x_{9}\left(x_{7}+x_{8}\right)\left(x_{6}+x_{7}+x_{8}\right)\left(x_{4}+x_{5}+x_{6}+x_{7}\right)\left(x_{2}+\cdots+x_{6}\right)}{x_{5}\left(x_{4}+x_{5}\right)\left(x_{3}+x_{4}+x_{5}\right)\left(x_{2}+x_{3}+x_{4}+x_{5}\right)\left(x_{1}+\cdots+x_{5}\right)},
\end{gathered}
$$

and therefore

$$
\operatorname{wt}(w)=\frac{x_{2} x_{9}^{2}\left(x_{8}+x_{9}\right)\left(x_{6}+x_{7}+x_{8}\right)\left(x_{4}+x_{5}+x_{6}+x_{7}\right)\left(x_{2}+\cdots+x_{6}\right)}{x_{1} x_{4} x_{8}\left(x_{3}+x_{4}\right)\left(x_{3}+x_{4}+x_{5}\right)\left(x_{2}+x_{3}+x_{4}+x_{5}\right)\left(x_{1}+\cdots+x_{5}\right)} .
$$

Example 5.3. Here are the values of $\operatorname{wt}(w)$ for $w$ in $\mathfrak{S}_{3}$ :

\begin{tabular}{|c|c|}
\hline$w$ & $\operatorname{wt}(w)$ \\
\hline \hline 123 & 1 \\
\hline 132 & $\frac{F^{2}[1]}{F[1]}=\frac{x_{3}}{x_{2}}$ \\
\hline 213 & $\frac{F[1]}{[1]}=\frac{x_{2}}{x_{1}}$ \\
\hline 231 & $\frac{F^{2}[1]}{[1]}=\frac{x_{3}}{x_{1}}$ \\
\hline 312 & $\frac{F[2] !}{[2] !}=\frac{F[2] F^{2}[1]}{[2] F[1]}=\frac{\left(x_{2}+x_{3}\right) x_{3}}{\left(x_{1}+x_{2}\right) x_{2}}$ \\
\hline 321 & $\frac{F[2] !}{[2] !} \frac{F[1]}{[1]}=\frac{F[2] F^{2}[1]}{[2][1]}=\frac{\left(x_{2}+x_{3}\right) x_{3}}{\left(x_{1}+x_{2}\right) x_{1}}$ \\
\hline
\end{tabular}

Four out of these six permutations $w$ in $\mathfrak{S}_{3}$, namely all except for $\{213,231\}$, are themselves recursively labelled forests when regarded as linear orders. For these four one can check that the value of $\operatorname{wt}(w)$ given in the table agrees with the product formula predicted by Theorem 1.1 . 
On the other hand, the two exceptions $\{213,231\}$ comprise $\mathcal{L}(P)$ for the recursively labelled forest poset $1>_{P} 2<_{P} 3$. One then checks from the values in the table that

$$
L(P)=\mathrm{wt}(213)+\mathrm{wt}(231)=\frac{x_{2}}{x_{1}}+\frac{x_{3}}{x_{1}}=\frac{x_{2}+x_{3}}{x_{1}}=\frac{F[2]}{[1]}
$$

which again agrees with the prediction of Theorem 1.1, namely

$$
\frac{[3] !}{F^{\min \left(P_{\geq} 1\right)-1}\left[h_{1}\right] \cdot F^{\min \left(P_{\geq}^{2}-1\right.}\left[h_{2}\right] \cdot F^{\min \left(P_{\geq} 3\right)-1}\left[h_{3}\right]}=\frac{[3] F[2] F^{2}[1]}{F^{0}[1] F^{0}[3] F^{2}[1]}=\frac{F[2]}{[1]} \text {. }
$$

For later use in Section 8, we explain how wt $(w)$ behaves under the specialization map $\operatorname{sp}_{q}$ from (1.4) which sends $x_{i}=F^{i}[1]$ to $q^{i-1}-q^{i}$. Note that

$$
\operatorname{sp}_{q} F^{i}[n]=\operatorname{sp}_{q}\left(x_{i}+x_{i+1}+\cdots+x_{i+n-1}\right)=q^{i-1}-q^{i+n-1}
$$

so that

$$
\operatorname{sp}_{q} \frac{F^{a}[n]}{F^{b}[m]}=q^{a-b} \frac{1-q^{n}}{1-q^{m}} .
$$

In particular, when $m=n$ one has

$$
\operatorname{sp}_{q} \frac{F^{a}[n]}{F^{b}[n]}=q^{a-b}
$$

and hence for a $k$-subset $S=\left\{i_{1}>i_{2}>\cdots>i_{k}\right\}$,

$$
\operatorname{sp}_{q} \operatorname{wt}(S)=\operatorname{sp}_{q} \prod_{j=1}^{k} \frac{F^{i_{j}-1}[j]}{F^{k-j}[j]}=q^{\sum_{j=1}^{k}\left(i_{j}-(k-j)-1\right)} .
$$

Corollary 5.4. For any permutation $w$ in $\mathfrak{S}_{n}$ one has $\operatorname{sp}_{q} \operatorname{wt}(w)=q^{\operatorname{inv}(w)}$.

Proof. Induct on $n$, with $n=0$ as a trivial base case. In the inductive step, if $w_{1}=k+1$ and $w=u \cdot a \cdot b$ is the parabolic factorization from (5.1), then

$$
\operatorname{inv}(w)=\operatorname{inv}(u)+\operatorname{inv}(a)+\operatorname{inv}(b) .
$$

Note that $\operatorname{inv}(b)=\operatorname{inv}(\hat{b})$. Also note that in (5.2), if one has $S(u)=\left\{i_{1}>\cdots>i_{k}\right\}$, then $\operatorname{inv}(u)=\sum_{j=1}^{k}\left(i_{j}-(k-j)-1\right)$ so that (5.7) implies

$$
q^{\operatorname{inv}(u)}=\operatorname{sp}_{q} \operatorname{wt}(S(u)) \text {. }
$$

Since by definition one has

$$
\mathrm{wt}(w)=\operatorname{wt}(S(u)) \cdot \operatorname{wt}(a) \cdot F^{k+1} \operatorname{wt}(\hat{b})
$$

the assertion of the corollary follows from (5.8), (5.9), together with the inductive hypothesis applied to $a$ and $\hat{b}$.

\section{The Weight of a Permutation, Via A SEARCh tree}

The goal of this section is to encode the recursive nature of the definition of the weight $\operatorname{wt}(w)$ for a permutation $w$ in a standard combinatorial data structure, an increasing binary search tree. Once this tree is computed, one no longer needs recursion to define $\operatorname{wt}(w)$.

Definition 6.1. (cf. Stanley [10, §1.3]) For any word $w=w_{1} \ldots w_{m}$ without repetition, define recursively its increasing binary tree $\mathrm{T}(w)$ as follows: 
- if $w$ is empty (i.e. $m=0$ ), then $\mathrm{T}(w)$ is the empty binary tree;

- else denote by $k$ the index of the smallest letter of $w$. Then $\mathrm{T}(w)$ is the binary tree whose root is labelled $w_{k}$, whose left subtree is $\mathrm{T}\left(w_{1} \ldots w_{k-1}\right)$ and whose right subtree is $\mathrm{T}\left(w_{k+1} \ldots w_{m}\right)$.

Now for a given permutation $w$, consider the tree $\mathrm{T}\left(w^{-1}\right)$. For each pair of labeled nodes $(\alpha, \beta)$ such that $\alpha$ occurs in the left subtree rooted at $\beta$, define a numerator polynomial $N(\alpha, \beta)$ and denominator polynomial $D(\alpha, \beta)$ by

$$
\begin{array}{lll}
D(\alpha, \beta) & :=x_{w(\beta)-1}+\cdots+x_{w(\beta)-\ell} & =F^{w(\beta)-\ell-1}[\ell] \\
N(\alpha, \beta) & :=F^{r+1}(D(\alpha, \beta)) & =F^{w(\beta)+r-\ell}[\ell]
\end{array}
$$

where $\ell:=\ell(\alpha, \beta)$ (resp. $r:=r(\alpha, \beta)$ ) is the number of nodes in the left (resp. right) subtree of $\beta$ whose label is larger or equal (resp. smaller) than $\alpha$. Note that since $\alpha$ is in the left subtree of $\beta$, one always has $\ell \geq 1$.

Example 6.2. For example, consider the permutation $w=541736829$. Its inverse is $w^{-1}=385216479$. The corresponding increasing tree $\mathrm{T}\left(w^{-1}\right)$ is therefore

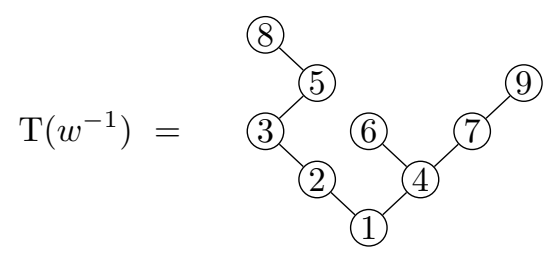

and the relevant pairs $(\alpha, \beta)$ and polynomials $N(\alpha, \beta), D(\alpha, \beta)$ are as follows:

\begin{tabular}{|c|c|c|c|c|c|c|}
\hline$\alpha$ & $\beta$ & $w(\beta)$ & $\ell$ & $r$ & $\begin{array}{c}N(\alpha, \beta) \\
:=F^{r+1} D(\alpha, \beta)\end{array}$ & $\begin{array}{c}D(\alpha, \beta) \\
:=F^{w(\beta)-\ell-1}([\ell])\end{array}$ \\
\hline \hline 2 & 1 & 5 & 4 & 0 & $x_{5}+x_{4}+x_{3}+x_{2}$ & $x_{4}+x_{3}+x_{2}+x_{1}$ \\
\hline 3 & 1 & 5 & 3 & 0 & $x_{5}+x_{4}+x_{3}$ & $x_{4}+x_{3}+x_{2}$ \\
\hline 5 & 1 & 5 & 2 & 1 & $x_{6}+x_{5}$ & $x_{4}+x_{3}$ \\
\hline 8 & 1 & 5 & 1 & 3 & $x_{8}$ & $x_{4}$ \\
\hline 3 & 2 & 4 & 3 & 0 & $x_{4}+x_{3}+x_{2}$ & $x_{3}+x_{2}+x_{1}$ \\
\hline 5 & 2 & 4 & 2 & 0 & $x_{4}+x_{3}$ & $x_{3}+x_{2}$ \\
\hline 8 & 2 & 4 & 1 & 0 & $x_{4}$ & $x_{3}$ \\
\hline 8 & 5 & 3 & 1 & 0 & $x_{3}$ & $x_{2}$ \\
\hline 6 & 4 & 7 & 1 & 0 & $x_{7}$ & $x_{6}$ \\
\hline
\end{tabular}

Proposition 6.3. For any permutation $w$, the weight of $w$ equals

$$
\operatorname{wt}(w)=\prod_{(\alpha, \beta)} \frac{N(\alpha, \beta)}{D(\alpha, \beta)},
$$

where the product is over $(\alpha, \beta)$ with $\alpha$ in the left subtree of $\mathrm{T}\left(w^{-1}\right)$ rooted at $\beta$.

Proof. Induct on $n$, with trivial base cases $n=0,1$. In the inductive step, let $L$ and $R$ be the left and right subtrees of the root of $\mathrm{T}\left(w^{-1}\right)$. Define $a, u$ and $\hat{b}$ as in Definition 5.1, Then

$$
\operatorname{wt}(w):=\operatorname{wt}(S(u)) \cdot \operatorname{wt}(a) \cdot F^{k+1}(\operatorname{wt}(\hat{b})) .
$$

Assume (6.1) holds for $w:=a$ or $w:=\hat{b}$; we wish to prove it holds for $w=u \cdot a \cdot b$. 
The tree $\mathrm{T}\left(a^{-1}\right)$ is obtained from $L$ by renumbering the labels to $\{1, \ldots, k\}$ keeping their relative order. Let $(\alpha, \beta)$ be two nodes of $L$ and $\left(\alpha^{\prime}, \beta^{\prime}\right)$ their renumbering in $\mathrm{T}\left(a^{-1}\right)$. It should be clear that

$$
\begin{aligned}
r(\alpha, \beta) & =r\left(\alpha^{\prime}, \beta^{\prime}\right), \\
\ell(\alpha, \beta) & =\ell\left(\alpha^{\prime}, \beta^{\prime}\right), \\
w(\beta) & =a\left(\beta^{\prime}\right) .
\end{aligned}
$$

As a consequence

$$
\mathrm{wt}(a)=\prod_{\left(\alpha^{\prime}, \beta^{\prime}\right)} \frac{N\left(\alpha^{\prime}, \beta^{\prime}\right)}{D\left(\alpha^{\prime}, \beta^{\prime}\right)}=\prod_{\substack{(\alpha, \beta) \\ \alpha, \beta \in L}} \frac{N(\alpha, \beta)}{D(\alpha, \beta)} .
$$

Similarly, the values of $r$ and $\ell$ also agree in $\mathrm{T}\left(\hat{b}^{-1}\right)$ and $R$, but the difference is that for two corresponding nodes $\beta \in R$ and $\beta^{\prime} \in \mathrm{T}\left(\hat{b}^{-1}\right)$, one has $w(\beta)=\hat{b}\left(\beta^{\prime}\right)+k+1$. It follows that

$$
F^{k+1}(\operatorname{wt}(\hat{b}))=\prod_{\left(\alpha^{\prime}, \beta^{\prime}\right)} F^{k+1}\left(\frac{N\left(\alpha^{\prime}, \beta^{\prime}\right)}{D\left(\alpha^{\prime}, \beta^{\prime}\right)}\right)=\prod_{\substack{(\alpha, \beta) \\ \alpha, \beta \in R}} \frac{N(\alpha, \beta)}{D(\alpha, \beta)}
$$

It therefore remains to show that $\mathrm{wt}(S(u))$ is exactly the product over pairs $(\alpha, \beta)$ with $\alpha=1$. Ordering decreasingly the labels $\left\{\alpha_{1}>\cdots>\alpha_{k}\right\}$ of $L$ which are also the elements of $S(u)$, one sees that

$$
\begin{aligned}
& \ell\left(\alpha_{j}, 1\right)=j, \\
& r\left(\alpha_{j}, 1\right)=\alpha_{j}-1-(k-j) .
\end{aligned}
$$

Since $w(1)=k+1$, one has

$$
\begin{aligned}
& D\left(\alpha_{j}, 1\right)=F^{k-j}[j], \\
& N\left(\alpha_{j}, 1\right)=F^{\alpha_{j}-1}[j] .
\end{aligned}
$$

Therefore

$$
\prod_{\alpha \in L}\left(\frac{N(\alpha, 1)}{D(\alpha, 1)}\right)=\prod_{j=1}^{f} \frac{F^{\alpha_{j}-1}[j]}{F^{k-j}[j]}=\frac{\prod_{j=1}^{k} F^{\alpha_{j}-1}[j]}{[k] !}=\operatorname{wt}(S(u)) .
$$

This proves that (6.1) holds for $w=u \cdot a \cdot b$.

Example 6.4. Continuing Example6.2 one sees that $a=4132$ so that $a^{-1}=2431$ and $b=57689$ so that $\hat{b}=2134$ and $\hat{b}^{-1}=2134$. As a consequence:

$$
\mathrm{T}\left(a^{-1}\right)=\underbrace{(4)}_{(1)} \mathrm{T}\left(\hat{b}^{-1}\right)=
$$

This gives a different way to view the assertion $\operatorname{sp}_{q} \mathrm{wt}(w)=q^{\mathrm{inv}(w)}$. of Corollary[5.4] Second proof of Corollary 5.4. Rephrasing Proposition 6.1 as

$$
\operatorname{wt}(w)=\prod_{(\alpha, \beta)} \frac{F^{r(\alpha, \beta)+1} D(\alpha, \beta)}{D(\alpha, \beta)}
$$


and bearing in mind (5.6), it suffices to check that

$$
\operatorname{inv}(w)=\sum_{(\alpha, \beta)}(r(\alpha, \beta)+1) .
$$

Let $(i<j)$ be an inversion of $w$, meaning that $w_{j}<w_{i}$. Looking at $w^{-1}$, this means that $j$ occurs to the left of $i$ in the word $w^{-1}=\left(w^{-1}(1), w^{-1}(2), \ldots, w^{-1}(n)\right)$. There are two possibilities:

- For all $r$ such that $w_{j}<r<w_{i}$ one has $i<w^{-1}(r)$.

In other words, in $w^{-1}$ all letters between $j$ and $i$ are bigger than $i$. By the construction of the tree $T=\mathrm{T}\left(w^{-1}\right)$, this implies that $j$ lies in the left subtree of $i$.

- There exists an $r$ such that $w_{j}<r<w_{i}$ and $w^{-1}(r)<i$.

In other words, one can find a letter smaller than $i$ lying between $j$ and $i$ in $w^{-1}$. Let $k$ be the minimal such letter:

$$
k:=\min \left\{w^{-1}(r) \mid w_{j}<r<w_{i}\right\} .
$$

By the construction of $T=\mathrm{T}\left(w^{-1}\right)$, the letter $k$ is the label of the only node $m$ of $T$ such that $j$ and $i$ are in the left and right subtrees of $m$. Therefore this $i$ counts for 1 in $r(\alpha, \beta)$ where $\alpha:=j$ and $\beta:=k$.

As a consequence, fixing $\alpha$, the sum $\sum_{\beta}(r(\alpha, \beta)+1)$ is exactly the number of $i<\alpha$ such that $w_{i}>w_{\alpha}$. This proves (6.6).

\section{Proof of Theorem 1.1}

For a recursively labelled forest $P$ on $\{1,2, \ldots, n\}$, we wish to prove equality of the two rational functions

$$
\begin{aligned}
L(P) & :=\sum_{w \in \mathcal{L}(P)} \mathrm{wt}(w), \\
H(P) & :=\frac{[n] !}{\prod_{i=1}^{n} F^{\min \left(P_{\geq i}\right)-1}\left[h_{i}\right]} .
\end{aligned}
$$

Proceed by induction on the following quantity: the sum of $n$ and the number of incomparable pairs $i, j$ in $P$. In the base case where this quantity is zero, in particular $n=0$, and the result is trivial. In the inductive step, there are two cases.

Case 1. There exist two elements $i, j$ having subtrees $P_{\geq i}, P_{\geq j}$ labelled by two contiguous intervals of integers, say

$$
\begin{aligned}
& P_{\geq i}=[r+1, r+s], \\
& P_{\geq j}=[r+s+1, r+s+t] .
\end{aligned}
$$

In this case, form the poset $P_{i<j}$ by taking the transitive closure of $P$ and the extra relation $i<j$. Defining $P_{j<i}$ similarly, one has the disjoint decomposition

$$
\mathcal{L}(P)=\mathcal{L}\left(P_{i<j}\right) \sqcup \mathcal{L}\left(P_{j<i}\right)
$$

since any $w$ in $\mathcal{L}(P)$ either has $i<_{w} j$ or $j<_{w} i$. Therefore

$$
L(P)=L\left(P_{i<j}\right)+L\left(P_{j<i}\right),
$$

and hence it remains to show

$$
H(P)=H\left(P_{i<j}\right)+H\left(P_{j<i}\right) .
$$


Because $P, P_{i<j}, P_{j<i}$ share the same size $n$, and share the same label sets on their subtrees $P_{\geq k}$ for $k \neq i, j$, the desired equality (7.2) is equivalent to checking

$$
\frac{1}{F^{r}[s] \cdot F^{r+s}[t]}=\frac{1}{F^{r}[s+t] \cdot F^{r+s}[t]}+\frac{1}{F^{r}[s] \cdot F^{r}[s+t]} \text {. }
$$

Over a common denominator, this amounts to checking

$$
F^{r}[s+t]=F^{r}[s]+F^{r+s}[t],
$$

which is immediated from the definition (1.2) of $[n]$.

Case 2. There are no such pairs of elements $i, j$ as in Case 1.

This means that $P$ is a recursively labelled binary tree, meaning that it has a minimum element, say $k+1$, and every element $i$ in $P$ is covered by at most one element $j<_{\mathbb{Z}} i$ and at most one element $j>_{\mathbb{Z}} i$. In particular, this means that the poset $P_{1}$ obtained by restricting $P$ to the values $[1, k]$ is again a recursively labelled binary tree. Similarly the restriction of $P$ to the values $[k+2, n]$ is obtained from some recursively labelled binary tree $P_{2}$ on values $[1, n-k-1]$ by adding $k+1$ to all of its vertex labels; denote this restriction $F^{k+1}\left(P_{2}\right)$.

One then calculates that

$$
\begin{aligned}
H(P) & =\frac{[n] !}{\prod_{i=1}^{n} F^{\min \left(P_{\geq i}\right)-1}\left[h_{i}\right]} \\
& =\frac{F[n-1] !}{\prod_{i \neq k+1} F^{\min \left(P_{\geq i}\right)-1}\left[h_{i}\right]} \\
& =\frac{F[k] !}{[k] !} \cdot F\left[\begin{array}{c}
n-1 \\
k
\end{array}\right] \cdot \frac{[k] !}{\prod_{i=1}^{k} F^{\min \left(P_{\geq i}\right)-1}\left[h_{i}\right]} \cdot \frac{F^{k+1}[n-1-k] !}{\prod_{i=k+2}^{n} F^{\min \left(P_{\geq i}\right)-1}\left[h_{i}\right]} \\
& =\frac{F[k] !}{[k] !} \cdot F\left[\begin{array}{c}
n-1 \\
k
\end{array}\right] \cdot H\left(P_{1}\right) \cdot F^{k+1} H\left(P_{2}\right) .
\end{aligned}
$$

It remains to show that $L(P)$ satisfies the same recurrence. Note that each $w$ in $\mathcal{L}(P)$ has $w_{1}=k+1$, because $k+1$ is the minimum element of $P$. Furthermore, when one decomposes $w=u \cdot a \cdot b$ as in the parabolic factorization (5.1) used to define $\operatorname{wt}(w)$, one finds that $a, \hat{b}$ lie in $\mathcal{L}\left(P_{1}\right), \mathcal{L}\left(P_{2}\right)$, respectively. Conversely, any such triple $(u, a, \hat{b})$, where $u$ is a shuffle of the sequences $(1,2, \ldots, k),(k+1, k+2, \ldots, n)$ having $u(1)=k+1$, gives rise to an element $w=u \cdot a \cdot b$ of $\mathcal{L}(P)$. Thus

$$
\begin{aligned}
L(P) & =\sum_{(u, a, \hat{b})} \operatorname{wt}(S(u)) \operatorname{wt}(a) F^{k+1} \operatorname{wt}(\hat{b}) \\
& =\frac{F[k] !}{[k] !}\left(\sum_{\substack{k-\text { subsets } \hat{S} \\
\text { of }\{1,2, \ldots, n-1\}}} F(\operatorname{wt}(\hat{S}))\right)\left(\sum_{a} \operatorname{wt}(a)\right)\left(\sum_{\hat{b}} F^{k+1} \operatorname{wt}(\hat{b})\right) \\
& =\frac{F[k] !}{[k] !} F\left[\begin{array}{c}
n-1 \\
k
\end{array}\right] \cdot L\left(P_{1}\right) \cdot F^{k+1} L\left(P_{2}\right)
\end{aligned}
$$

using (5.4) and (4.3).

Thus in both cases, $L(P)$ and $H(P)$ satisfy the same recurrence, concluding the proof of Theorem 1.1 . 


\section{Specializing to the FORMUla of BJÖRner AND WACHS}

It is now easy to deduce Björner and Wachs' identity (1.1) as the $q$-specialization of Theorem 1.1. one has from Corollary 5.4 that

$$
\operatorname{sp}_{q} L(P)=\sum_{w \in \mathcal{L}(P)} q^{\operatorname{inv}(w)},
$$

while the right side $H(P)$ of Theorem 1.1 has $q$-specialization

$$
\begin{aligned}
\operatorname{sp}_{q} H(P) & =\operatorname{sp}_{q} \prod_{i=1}^{n} \frac{F^{i-1}[n-i+1]}{F^{\min \left(P_{\geq i}\right)-1}\left[h_{i}\right]} \\
& =q^{\sum_{i=1}^{n}\left(i-\min \left(P_{\geq i}\right)\right)} \prod_{i=1}^{n} \frac{1-q^{n-i+1}}{1-q^{h_{i}}} \quad \text { using (5.5) } \\
& =q^{\operatorname{inv}(P)} \frac{[n] !_{q}}{\prod_{i=1}^{n}\left[h_{i}\right]}
\end{aligned}
$$

where the last equality used the following fact: since $P$ is a recursively labelled forest, for each $i$, the quantity $i-\min \left(P_{\geq i}\right)$ counts the contribution to $\operatorname{inv}(P)$ coming from the pairs $(i, j)$ where $j$ lies in $P_{\geq i}$.

\section{Algebra morphisms}

Theorem 1.1 has an interesting rephrasing in terms of a $\mathbb{Q}$-linear map from the ring of free quasisymmetric functions $\mathcal{F} \mathcal{Q S}$ ym (or Malvenuto-Reutenauer algebra) into a certain target ring. We define these objects here.

Definition 9.1. Recall from 9 that the algebra $\mathcal{F} \mathcal{Q S} y m$ has $\mathbb{Q}$-basis elements

$$
\left\{\mathbf{F}_{w}: w \in \bigsqcup_{n \geq 0} \mathfrak{S}_{n}\right\},
$$

with multiplication defined $\mathbb{Q}$-bilinearly as follows: for $a, b$ lying in $\mathfrak{S}_{k}, \mathfrak{S}_{\ell}$ one has

$$
\mathbf{F}_{a} \cdot \mathbf{F}_{b}:=\sum_{w} \mathbf{F}_{w}
$$

where $w$ runs through all shuffles of the words

$$
\begin{aligned}
a & =\left(a_{1}, \ldots, a_{k}\right), \text { and } \\
F^{k}(b) & :=\left(b_{1}+k, \ldots, b_{\ell}+k\right) .
\end{aligned}
$$

One of the original motivations for introducing the ring $\mathcal{F} \mathcal{Q S} y m$ is the following. Define for each poset $P$ the element

$$
\mathbf{F}_{P}:=\sum_{w \in \mathcal{L}(P)} \mathbf{F}_{w}
$$

in $\mathcal{F} Q \mathcal{S} y m$. Then for two posets $P, Q$ on elements $\{1,2, \ldots, k\},\{1,2, \ldots, \ell\}$, respectively, one has in $\mathcal{F} \mathcal{Q S}$ ym that

$$
\mathbf{F}_{P} \cdot \mathbf{F}_{Q}=\mathbf{F}_{P \sqcup F^{k}(Q)},
$$

where $P \sqcup F^{k}(Q)$ denotes the poset on $\{1,2, \ldots, k+\ell\}$ which is the disjoint union of $P$ with the poset $F^{k}(Q)$ on $\{k+1, k+2, \ldots, k+\ell\}$ obtained by adding $k$ to each label in $Q$. 
Definition 9.2. Let the semigroup $\mathbb{N}=\left\{1, F, F^{2}, \ldots\right\}$ act on the rational functions $\mathbb{Q}(\mathbf{x})=\mathbb{Q}\left(x_{1}, x_{2}, \ldots\right)$ via the Frobenius map as before: $F\left(x_{i}\right)=x_{i+1}$. Then define the skew semigroup algebra $\mathbb{Q}(\mathbf{x}) \# \mathbb{N}$ to be the free $\mathbb{Q}(\mathbf{x})$-module on basis $\left\{1, u, u^{2}, \ldots\right\}$, with multiplication defined $\mathbb{Q}$-linearly by

$$
f(\mathbf{x}) u^{k} \cdot g(\mathbf{x}) u^{\ell}=\left(f(\mathbf{x}) F^{k}(g(\mathbf{x}))\right) u^{k+\ell} .
$$

One of our motivations for introducing $\mathbb{Q}(\mathbf{x}) \# \mathbb{N}$ is that, in addition to its $\mathbb{Q}(\mathbf{x})$-basis $\left\{1, u, u^{2}, \ldots\right\}$, it also has a $\mathbb{Q}(\mathbf{x})$-basis of divided powers $\left\{1, u^{(1)}, u^{(2)}, \ldots\right\}$, where

$$
u^{(n)}:=\frac{1}{[n] !} u^{n},
$$

and this basis has our binomial coefficients as multiplicative structure constants:

$$
u^{(k)} \cdot u^{(\ell)}=\left[\begin{array}{c}
k+\ell \\
k
\end{array}\right] u^{(k+\ell)} .
$$

Definition 9.3. Define the $\mathbb{Q}$-linear map

$$
\begin{array}{rll}
\mathcal{F} \mathcal{Q S} y m & \stackrel{\phi_{\text {in }}}{\longrightarrow} & \mathbb{Q}(\mathbf{x}) \# \mathbb{N} \\
\mathbf{F}_{w} & \longmapsto & \frac{\mathrm{w}(w)}{[n] !} u^{n}=\operatorname{wt}(w) \cdot u^{(n)}
\end{array}
$$

for $w$ in $\mathfrak{S}_{n}$. Note that

$$
\phi_{\text {inv }}\left(\mathbf{F}_{P}\right)=L(P) \cdot u^{(n)} .
$$

This $\mathbb{Q}$-linear map $\phi_{\text {inv }}$ turns out not to be an algebra morphism. E.g., one can check via explicit computations that

$$
\begin{aligned}
\phi_{\text {inv }}\left(\mathbf{F}_{1} \cdot \mathbf{F}_{213}\right) & =\phi_{\text {inv }}\left(\mathbf{F}_{1324}\right)+\phi_{\text {inv }}\left(\mathbf{F}_{3124}\right)+\phi_{\text {inv }}\left(\mathbf{F}_{3214}\right)+\phi_{\text {inv }}\left(\mathbf{F}_{3241}\right) \\
& \neq \phi_{\text {inv }}\left(\mathbf{F}_{1}\right) \cdot \phi_{\text {inv }}\left(\mathbf{F}_{213}\right) .
\end{aligned}
$$

However, the import of Theorem 1.1 is that $\phi_{\mathrm{inv}}$ becomes an algebra morphism when restricted to an appropriate subalgebra of $\mathcal{F} \mathcal{Q S} y m$.

Definition 9.4. Recall from 8 , that the Loday-Ronco algebra of binary trees $\mathcal{P B T}$ can be defined as the subalgebra of $\mathcal{F} \mathcal{Q S} y m$ spanned by all $\left\{\mathbf{F}_{P}\right\}$ as $P$ runs through all recursively labelled forests.

Proposition 9.5. When restricted from $\mathcal{F} Q \mathcal{S} y m$ to $\mathcal{P B T}$, the map $\phi_{\text {inv }}$ becomes an algebra homomorphism $\mathcal{P} \mathcal{B} \mathcal{T} \stackrel{\phi_{\text {inx }}}{\longrightarrow} \mathbb{Q}(\mathbf{x}) \# \mathbb{N}$.

Proof. It is easy to check that the product formula $H(P)$ defined in (7.1) for a recursively labelled forest $P$ satisfies

$$
H\left(P \sqcup F^{k} Q\right)=\left[\begin{array}{c}
k+\ell \\
k
\end{array}\right] H(P) \cdot F^{k} H(Q) .
$$

Hence for recursively labelled forests $P, Q$ of sizes $k, \ell$, one has

$$
\begin{aligned}
\phi_{\text {inv }}\left(\mathbf{F}_{P} \cdot \mathbf{F}_{Q}\right) & =\phi_{\text {inv }}\left(\mathbf{F}_{P \sqcup F^{k} Q}\right) & & \text { by (9.2) } \\
& =L\left(P \sqcup F^{k} Q\right) \cdot u^{(k+\ell)} & & \text { by (9.4) } \\
& =H\left(P \sqcup F^{k} Q\right) \cdot u^{(k+\ell)} & & \text { by Theorem } 1.1] \\
& =\left[\begin{array}{c}
k+\ell \\
k
\end{array}\right] H(P) \cdot F^{k} H(Q) \cdot u^{(k+\ell)} & & \text { by (9.5) } \\
& =H(P) u^{(k)} \cdot H(Q) u^{(\ell)} & & \text { by (9.3) } \\
& =L(P) u^{(k)} \cdot L(Q) u^{(\ell)} & & \text { by Theorem } 1.1] \\
& =\phi_{\text {inv }}\left(\mathbf{F}_{P}\right) \cdot \phi_{\text {inv }}\left(\mathbf{F}_{Q}\right) & & \text { by (9.4). }
\end{aligned}
$$


Remark 9.6. This twisted semigroup algebra $\mathbb{Q}(\mathbf{x}) \# \mathbb{N}$ also appears implicitly in the theory of $P$-partitions, as the target of a different map $\phi_{\text {maj }}: \mathcal{F} \mathcal{Q S} y m \rightarrow \mathbb{Q} \# \mathbb{N}$, which is an algebra morphism. This is related to a recent multivariate generalization of Björner and Wachs' other "maj" q-hook formula for forests. We describe both connections briefly here.

For a poset $P$ on $\{1,2, \ldots, n\}$, a $P$-partition (see [10, $\S 4.5$ and 7.19]) is a weakly order-reversing function $f: P \rightarrow \mathbb{N}$ (meaning $i<_{P} j$ implies $f(i) \geq f(j)$ ) which is strictly decreasing along descent covering relations: whenever $j$ covers $i$ in $P$ and $i>_{\mathbb{Z}} j$ then $f(i)>f(j)$. Define their generating function $\gamma(P, \mathbf{x}):=\sum_{f} \mathbf{x}^{f}$ where here $f$ runs over all $P$-partitions, and $\mathbf{x}^{f}:=x_{1}^{f(1)} \cdots x_{n}^{f(n)}$. The relevant algebra morphism is defined $\mathbb{Q}$-bilinearly as follows:

$$
\begin{aligned}
\mathcal{F} \mathcal{Q S} y m & \stackrel{\phi_{\text {maj }}}{\longrightarrow} \mathbb{Q} \# \mathbb{N} \\
\mathbf{F}_{w} & \longmapsto \gamma(w, \mathbf{x}) \cdot u^{n} .
\end{aligned}
$$

The main proposition on $P$-partitions [10, Theorem 4.54] asserts that

$$
\gamma(P, \mathbf{x})=\sum_{w \in \mathcal{L}(P)} \gamma(w, \mathbf{x})
$$

or equivalently,

$$
\phi_{\text {maj }}\left(\mathbf{F}_{P}\right)=\gamma(P, \mathbf{x}) u^{n} .
$$

This then shows that $\phi_{\text {maj }}$ is an algebra morphism, since for any posets $P, Q$ on $[1, k]$ and $[1, \ell]$, one has

$$
\begin{aligned}
\phi_{\mathrm{maj}}\left(\mathbf{F}_{P} \cdot \mathbf{F}_{Q}\right) & =\phi_{\mathrm{maj}}\left(\mathbf{F}_{P \sqcup F^{k}(Q)}\right) \\
& =\gamma\left(P \sqcup F^{k}(Q), \mathbf{x}\right) u^{k+\ell} \\
& =\gamma(P, \mathbf{x}) \cdot F^{k}(\gamma(Q, \mathbf{x})) u^{k+\ell} \\
& =\gamma(P, \mathbf{x}) u^{k} \cdot \gamma(Q, \mathbf{x}) u^{\ell} \\
& =\phi_{\text {maj }}\left(\mathbf{F}_{P}\right) \cdot \phi_{\text {maj }}\left(\mathbf{F}_{Q}\right) .
\end{aligned}
$$

The Björner-Wachs maj formula arises when $P$ is a dual forest, that is, every element $i$ in $P$ is covered by at most one other element $j$; say that $i$ is a descent of $P$ if in addition $i>_{\mathbb{Z}} j$. Let $\operatorname{Des}(P)$ denote the set of descents of $P$, and $\operatorname{maj}(P):=\sum_{i \in \operatorname{Des}(P)} i$. In particular, permutations $w=\left(w_{1}, \ldots, w_{n}\right)$ considered as linear orders are dual forests, and for them one has maj $(w)=\sum_{i: w_{i}>w_{i+1}} i$. For any dual forest $P$, note that the subtree rooted at $i$ is $P_{\leq i}$, and again denote its cardinality by $h_{i}$. The Björner-Wachs maj formula asserts the following.

Theorem. ([1, Theorem 1.2]) Any dual forest $P$ on $\{1,2, \ldots, n\}$ has

$$
\sum_{w \in \mathcal{L}(P)} q^{\operatorname{maj}(w)}=q^{\operatorname{maj}(P)} \frac{[n] !_{q}}{\prod_{i=1}^{n}\left[h_{i}\right]_{q}} .
$$

The following generalization was observed recently in [2]: 
Theorem. For any dual forest $P$ on $\{1,2, \ldots, n\}$, one has

$$
\gamma(P, \mathbf{x}):=\frac{\prod_{i \in \operatorname{Des}(P)} \mathbf{x}_{P_{\leq i}}}{\prod_{i=1}^{n}\left(1-\mathbf{x}_{P_{\leq i}}\right)}
$$

where $\mathbf{x}_{S}:=\prod_{j \in S} x_{j}$, so that (9.6) becomes

$$
\sum_{w \in \mathcal{L}(P)}\left(\frac{\prod_{i \in \operatorname{Des}(w)} x_{w_{1}} \cdots x_{w_{i}}}{\prod_{i=1}^{n}\left(1-x_{w_{1}} \cdots x_{w_{i}}\right)}\right)=\frac{\prod_{i \in \operatorname{Des}(P)} x_{P_{\leq i}}}{\prod_{i=1}^{n}\left(1-x_{P_{\leq i}}\right)} .
$$

The Björner-Wachs maj formula is immediate upon specializing $x_{i}=q$ in (9.9):

$$
\sum_{w \in \mathcal{L}(P)} \frac{q^{\operatorname{maj}(w)}}{(1-q)\left(1-q^{2}\right) \cdots\left(1-q^{n}\right)}=\frac{q^{\operatorname{maj}(P)}}{\prod_{i=1}^{n}\left(1-q^{h_{i}}\right)} .
$$

Remark 9.7. The maps $\phi_{\text {inv }}, \phi_{\text {maj }}: \mathcal{F} \mathcal{Q S} y m \rightarrow \mathbb{Q}(\mathbf{x}) \# \mathbb{N}$ are reminiscent of the formalism of moulds discussed by Chapoton, Hivert, Novelli and Thibon 3, but we have not yet found a deeper connection.

One might also hope that the $(q, t)$-specializations $\operatorname{sp}_{q, t} L(P)$ for recursively labelled binary trees $P$ can be given a representation-theoretic interpretation, similar to the discussion in Section 2, but related to $q$-analogues of the indecomposable projective modules for the algebras whose existence is conjectured by Hivert, Novelli and Thibon in [4, §5.2]. At the moment this is purely speculative.

\section{REFERENCES}

[1] A. Björner and M.L. Wachs, q-hook length formulas for forests. J. Combin. Theory Ser. A 52 (1989), no. 2, 165-187.

[2] A. Boussicault, V. Feray, and V. Reiner, Rational function identities and valuations on cones. In preparation, 2010.

[3] F. Chapoton, F. Hivert, J.-C. Novelli, J.-Y. Thibon, An operational calculus for the mould operad. Int. Math. Res. Not. IMRN (2008), no. 9, Art. ID rnn018, 22 pp

[4] F. Hivert, J.-C. Novelli, J.-Y. Thibon, The algebra of binary search trees. Theoret. Comput. Sci. 339 (2005), no. 1, 129-165.

[5] F. Hivert, J.-C. Novelli, J.-Y. Thibon, Trees, functional equations, and combinatorial Hopf algebras. European J. Combin. 29 (2008), no. 7, 1682-1695

[6] T.J. Hewett, Modular invariant theory of parabolic subgroups of $\mathrm{GL}_{n}\left(F_{q}\right)$ and the associated Steenrod modules. Duke Math. J. 82 (1996), no. 1, 91-102.

[7] D.E. Knuth, Sorting and searching, in "The art of computer programming, Vol. 3" AddisonWesley, Reading MA, 1973.

[8] J.-L. Loday and M.O. Ronco, Hopf algebra of the planar binary trees. Adv. Math. 139 (1998), no. 2, 293-309.

[9] C. Malvenuto and C. Reutenauer, Duality between quasi-symmetric functions and the Solomon descent algebra. J. Algebra 177 (1995), 967-982.

[10] R.P. Stanley, Enumerative Combinatorics, Volumes 1 and 2. Cambridge Studies in Advanced Mathematics, 49 and 62. Cambridge University Press, Cambridge, 1997 and 1999.

[11] V. Reiner and D. Stanton, $(q, t)$-analogues and $G L_{n}\left(\mathbb{F}_{q}\right)$. arXiv:0804.3074 to appear in J. Algebraic. Combin.

E-mail address: florent.hivert@univ-rouen.fr

Litis, Université de Rouen, Avenue de l’Universit'e, 76801 Saint Étienne du Rouvray, FRANCE

E-mail address: reiner@math.umn.edu

School of Mathematics, University of Minnesota, Minneapolis, MN 55455, USA 